\title{
Magnetic fields and radio emission processes in maser-emitting planetary nebulae
}

\author{
L. Uscanga ${ }^{1}$, J. F. Gómez ${ }^{2}$, J. A. Green ${ }^{3}$, O. Suárez ${ }^{4}$, H.-H. Qiao ${ }^{5}$,

 \\ P. Boumis ${ }^{7}$ \\ ${ }^{1}$ University of Guanajuato, Mexico; email: lucero@astro.ugto.mx; \\ ${ }^{2}$ IAA-CSIC, Spain; ${ }^{3}$ CASS, Australia; ${ }^{4}$ OCA, France; ${ }^{5}$ NTSC, CAS, China; \\ ${ }^{6}$ ICRAR, CU, Australia; ${ }^{7}$ IAASARS, NOA, Greece
}

\begin{abstract}
We present polarimetric observations of the 4 ground-state transitions of $\mathrm{OH}$, toward a sample of maser-emitting planetary nebulae (PNe) using the Australia Telescope Compact Array. This sample includes confirmed $\mathrm{OH}$-emitting $\mathrm{PNe}$, confirmed and candidate $\mathrm{H}_{2} \mathrm{O}$-maseremitting PNe. Polarimetric observations provide information related to the magnetic field of these sources. Maser-emitting PNe are very young PNe and magnetic fields are a key ingredient in the early evolution and shaping process of PNe. Our preliminary results suggest that magnetic field strengths may change very rapidly in young $\mathrm{PNe}$.
\end{abstract}

Keywords. magnetic fields - masers - polarization - stars: AGB and post-AGB - planetary nebulae: general.

\section{Introduction}

Previous polarimetric studies of a few OHPNe with single-dish telescopes shown that circularly polarized $\mathrm{OH}$ features are common in OHPNe, but the detection of Zeeman pairs is far more elusive (Szymczak \& Gérard 2004, Wolak et al. 2012, Gonidakis et al. 2014). However, new interferometric observations have shown more promising candidates. The only OHPN in which Zeeman splitting has been clearly detected is IRAS 16333-4807 at $1720 \mathrm{MHz}$ (Qiao et al. 2016), with a derived magnetic strength of $\simeq 11 \mathrm{mG}$. Another possible Zeeman pair has been reported in K3-35 at $1665 \mathrm{MHz}$ (Gómez et al. 2009), giving $B \simeq 0.9 \mathrm{mG}$. Both objects are probably among the youngest $\mathrm{PNe}$, since both are $\mathrm{OH}$ and $\mathrm{H}_{2} \mathrm{O}$ maser emitters. Gómez et al. (2016) presented full-polarizations observations of the $4 \mathrm{OH}$ transitions, toward 5 confirmed and 1 candidate OHPNe. We detected significant circular and linear polarization, in 4 and 2 objects, respectively. Possible Zeeman pairs were seen in JaSt 23 and IRAS 17393-2727, with magnetic field strengths $0.8-24 \mathrm{mG}$.

\section{Observations and Results}

New observations were carried out with the ATCA, in its 6A configuration, on 2017 February 13-14. We obtained data in full polarization (two linear polarizations and their corresponding cross-polarizations). Broadband continuum data cover a bandwidth of 2 $\mathrm{GHz}$, centered at 2.1, 5.5 and $9 \mathrm{GHz}$. We also observed the $\mathrm{OH}$ ground-level transitions of rest frequency $1612,1665,1667$, and $1720 \mathrm{MHz}$ with a spectral resolution of $0.5 \mathrm{kHz}$ $\left(\simeq 0.09 \mathrm{~km} \mathrm{~s}^{-1}\right)$. For these observations, our main targets were IRAS 16333-4807 (I16333), IRAS 17393-2727 (I17393), and IRAS 18061-2505 (I18061). Radio continuum emission is detected in all three sources. We detected $\mathrm{OH}$ maser emission in I16333 and I17393, while no $\mathrm{OH}$ maser emission was detected $(<0.06 \mathrm{Jy})$ in I18061. 

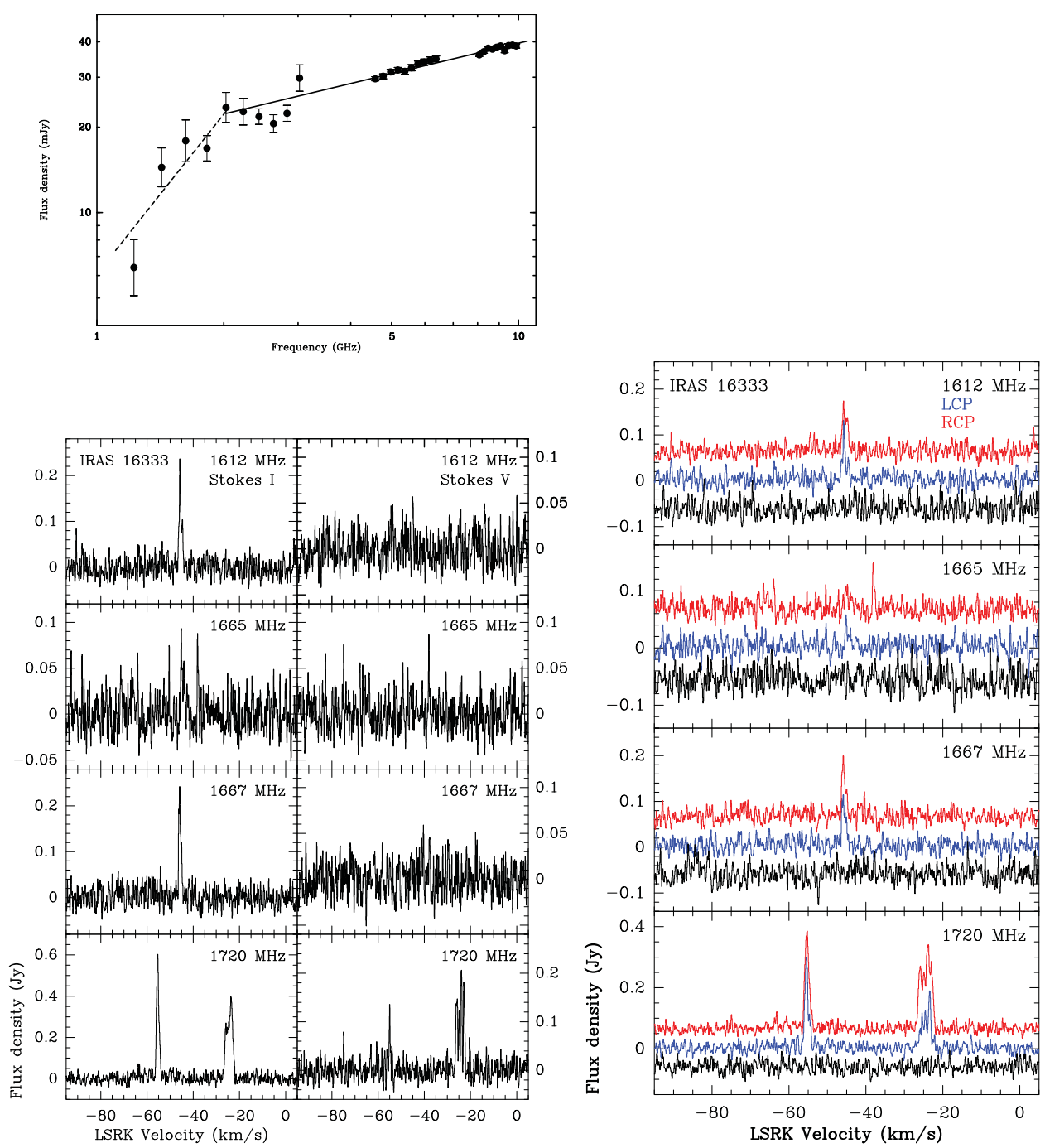

Figure 1. Top left: Flux density of $\mathrm{I} 16333$ as a function of frequency. The emission at $<2 \mathrm{GHz}$ is optically thick (spectral index $\simeq 1.87)$ and partially thick $(\simeq 0.36)$ at higher frequencies. Bottom left: OH maser spectra in Stokes I and V. Bottom right: circular (red and blue) and linear (black) polarizations.

Here we present preliminary results of I16333 (Fig. 1). The flux densities are consistent with the values measured previously by Qiao et al. (2016). These spectra do not show a clear evidence of Zeeman pairs as in previous observations (Qiao et al. 2016). Our results may indicate a fast change in the magnetic field strength within a few years.

\section{References}

Gómez, J. F., Uscanga, L., Green, J. A., et al. 2016, MNRAS, 461, 3259

Gómez, Y., Tafoya, D., Anglada, G., et al. 2009, ApJ, 695, 930

Gonidakis, I., Chapman, J. M., Deacon, R. M., \& Green, A. J. 2014, MNRAS, 443, 3819

Qiao, H.-H., Walsh, A. J., Gómez, J. F., et al. 2016, ApJ, 817, 37

Szymczak, M. \& Gérard, E. 2004, A\&\&A, 423, 209

Wolak, P., Szymczak, M., \& Gérard, E. 2012, A\&A, 537, A5 